\title{
Real-time determination of intracellular oxygen in bacteria using a genetically encoded FRET-based biosensor
}

\author{
Janko Potzkei ${ }^{1 \dagger}$, Martin Kunze ${ }^{2 \dagger}$, Thomas Drepper ${ }^{1 * \dagger}$, Thomas Gensch ${ }^{3}$, Karl-Erich Jaeger ${ }^{1 *}$ and Jochen Büchs ${ }^{2}$
}

\begin{abstract}
Background: Molecular oxygen $\left(\mathrm{O}_{2}\right)$ is one of the key metabolites of all obligate and facultative aerobic pro- and eukaryotes. It plays a fundamental role in energy homeostasis whereas oxygen deprivation, in turn, broadly affects various physiological and pathophysiological processes. Therefore, real-time monitoring of cellular oxygen levels is basically a prerequisite for the analysis of hypoxia-induced processes in living cells and tissues.

Results: We developed a genetically encoded Förster resonance energy transfer (FRET)-based biosensor allowing the observation of changing molecular oxygen concentrations inside living cells. This biosensor named FluBO (fluorescent protein-based biosensor for oxygen) consists of the yellow fluorescent protein (YFP) that is sensitive towards oxygen depletion and the hypoxia-tolerant flavin-binding fluorescent protein (FbFP). Since $\mathrm{O}_{2}$ is essential for the formation of the YFP chromophore, efficient FRET from the FbFP donor domain to the YFP acceptor domain only occurs in the presence but not in the absence of oxygen. The oxygen biosensor was used for continuous real-time monitoring of temporal changes of $\mathrm{O}_{2}$ levels in the cytoplasm of Escherichia coli cells during batch cultivation.

Conclusions: FluBO represents a unique FRET-based oxygen biosensor which allows the non-invasive ratiometric readout of cellular oxygen. Thus, FluBO can serve as a novel and powerful probe for investigating the occurrence of hypoxia and its effects on a variety of (patho)physiological processes in living cells.
\end{abstract}

\section{Background}

Non-invasive detection of intracellular $\mathrm{O}_{2}$ is of particular importance since it is one of the key metabolites of obligate and facultative aerobic organisms. Cellular $\mathrm{O}_{2}$ is a prominent indicator for oxygen-dependent metabolic activities, such as aerobic respiration or oxygen dependent synthesis and degradation of cellular components $[1,2]$. In addition, various biological, pathological and biotechnological processes are controlled by $\mathrm{O}_{2}$ limitation, including biofilm formation and host-pathogen interactions [3-7], hypoxia induced inflammatory processes [8], tumor pathophysiology [9-12] as well as microbial

\footnotetext{
* Correspondence: t.drepper@fz-juelich.de; k.-e.jaeger@fz-juelich.de + Contributed equally

${ }^{1}$ Institute of Molecular Enzyme Technology, Heinrich-Heine-University Duesseldorf, Juelich Research Center, Wilhelm-Johnen-Straße, D-52425 Juelich, Germany

Full list of author information is available at the end of the article
}

fermentation processes used for bioremediation and the production of food, feed and biofuels [13-16].

To date, different minimally invasive fluorescence and phosphorescence based $\mathrm{O}_{2}$-sensitive probes have been developed for imaging molecular oxygen in cells and tissues. Among them, platinum(II)-porphyrin dyes are widely used for analyzing hypoxia-induced responses of mammalian cells [17-19]. Alternatively, the green fluorescent protein (GFP) and its variants can be applied as genetically encoded intracellular probes that are specifically expressed and can be selectively targeted within defined cells and tissues. In this context, at least two 'passive' GFP-based oxygen sensors have been developed for estimating intracellular oxygen levels in E. coli. Here, GFP was applied as a reporter protein expressed under control of oxygen-responsive $E$. coli promoters [20,21]. Additionally, oxygen sensitive photoactivation of GFPmediated red fluorescence was applied for in vivo imaging of oxygen in mammalian cells and organs [22-24].
C Biomed Central

C 2012 Potzkei et al; licensee BioMed Central Ltd. This is an Open Access article distributed under the terms of the Creative Commons Attribution License (http://creativecommons.org/licenses/by/2.0), which permits unrestricted use, distribution, and reproduction in any medium, provided the original work is properly cited. 
Remarkably, molecular oxygen can currently not be analyzed in vivo by genetically encoded FRET-based biosensors, although these biosensors constitute one of the most widespread classes of fluorescent molecular probes used for the non-invasive quantitative analysis of intracellular compounds including $\mathrm{Ca}^{2+}, \mathrm{Zn}^{2+}, \mathrm{Cl}^{-}, \mathrm{pH}, \mathrm{H}_{2} \mathrm{O}_{2}$, ATP, maltose, sucrose, ribose and glucose [25-27]. With respect to oxygen sensing, however, GFP-like proteins, which are commonly used as donor and acceptor domains of FRET biosensors, exhibit a major drawback: Their autocatalytic chromophore synthesis strictly depends on the presence of molecular oxygen $[28,29]$ and thus the fluorescence signal intensity basically does not reflect the amount of synthesized reporter protein [30]. Therefore, GFP and its color variants can not solely be used as fluorescent biosensor domains for accurate $\mathrm{O}_{2}$ determination.

Recently, we developed a novel class of fluorescent proteins which carry flavin mononucleotide (FMN) as chromophore [31]. In contrast to GFP-like FPs, the fluorescence signal of these FMN-based fluorescent proteins (FbFP) is independent of cellular oxygen and thus FbFP can be used as quantitative in vivo real-time reporter protein under aerobic as well as anaerobic conditions $[30,31]$. Here, we report the construction and application of the first genetically encoded FRET-based biosensor for oxygen named FluBO which consists of the oxygen-insensitive FbFP donor domain and the hypoxia-sensitive enhanced yellow fluorescent protein (YFP) acceptor domain. We further show that its FRET efficiency dynamically responds to changing $\mathrm{O}_{2}$ values in living bacterial cells.

\section{Results}

\section{Construction and in vitro characterization of FluBO}

The in vivo fluorescence of YFP, in contrast to FbFP, strictly depends on intracellular oxygen [30]. This observation prompted us to test if a YFP-FbFP fusion can be used as a ratiometric FRET-based biosensor for self-referenced determination of molecular oxygen levels in living cells. The canonical intramolecular FRET-based biosensors are generally fusion proteins consisting of two fluorescent domains with different chromophores that show spectral overlap between the absorption wavelengths of the acceptor domain and the emission wavelengths of the donor domain $[32,33]$. Therefore, we constructed a recombinant gene (Figure 1A, Additional file 1) encoding the oxygen biosensor which consists of the $\mathrm{N}$-terminal YFP acceptor domain and the C-terminal FbFP donor domain interconnected by a small peptide linker with a thrombin protease cleavage site. Since the enhanced cyan FP (CFP) has most widely been used together with YFP in a tandem FRET biosensor design $[32,34]$, we initially tested whether CFP can be functionally substituted by FbFP as the FRET donor domain. For that purpose, YFP and FbFP as well as the YFP-FbFP fusion protein were separately expressed, purified and their spectral characteristics were analyzed. Figure 1B shows the absorption and emission spectra of FbFP and YFP, respectively. FbFP exhibited its typical absorption spectrum ranging from near UV to blue light with $\lambda_{\max }$ at $450 \mathrm{~nm}$, which is characteristic for the FMN chromophore [31]. As required for suitable FRET pairs, the fluorescence emission spectrum of FbFP $\left(\lambda_{\max }=495\right.$ $\mathrm{nm})$ substantially overlaps with the YFP absorption spectrum $\left(\lambda_{\max }=512 \mathrm{~nm}\right)$ whereas the emission peaks of FbFP and YFP are sufficiently separated (YFP $\lambda_{\max }=528$ $\mathrm{nm})$. To minimize direct excitation of the acceptor at the donor excitation wavelength, two-dimensional wavelength scans of FbFP and YFP have been carried out. As demonstrated in Figure 1C, an excitation wavelength of $380 \mathrm{~nm}$ results in a bright FbFP fluorescence and a very low excitation of YFP fluorescence. Beside its spectral characteristics, the quantum yield $(\mathrm{QY})$ of FbFP [31] $(\mathrm{QY}=0.39)$ resembles that of CFP [35] (QY $=0.36)$ indicating that FbFP may be suitable as a FRET donor for YFP.

FRET is a phenomenon of nonradiative energy transfer between the chromophores of the donor and acceptor domain, which can be observed either by a change of (i) ratio of fluorescence intensities emitted by each domain or (ii) lifetime of donor fluorescence. Efficient intramolecular FRET thus results in a decrease of donor fluorescence intensity and of excited-state lifetime. To demonstrate that the YFP-FbFP fusion protein forms a functional FRET pair we first analyzed its fluorescence properties in vitro. Fluorescence emission spectra (Figure 1D) of FbFP, YFP and FluBO, respectively, recorded with an excitation wavelength of $380 \mathrm{~nm}$ clearly demonstrate that the FbFP fluorescence (green) is largely decreased by efficient energy transfer leading to a dominant fluorescence of the YFP acceptor domain within the fusion protein (red). In contrast, fluorescence intensity of the plain acceptor was low at the same excitation wavelength (yellow). Protease cleavage of the two fluorescent domains resulted in distances much larger than the Förster radius between the donor and acceptor domain and thus in the loss of FRET coupling, which is reflected by a dequenching of the donor FbFP and a dramatic decrease of YFP fluorescence (see Additional file 2).

\section{In vivo functionality of FluBO}

Next, FluBO was tested as a FRET-based ratiometric oxygen probe in living cells. Initially, we analyzed the FbFP emission of FluBO in E. coli by fluorescence lifetime imaging (FLIM) under aerobic conditions leading to complete maturation of the YFP (that is, the FluBO acceptor domain) chromophore. Figure 2A shows E. coli cells expressing either FbFP or FluBO color-coded according to their average fluorescence lifetimes 

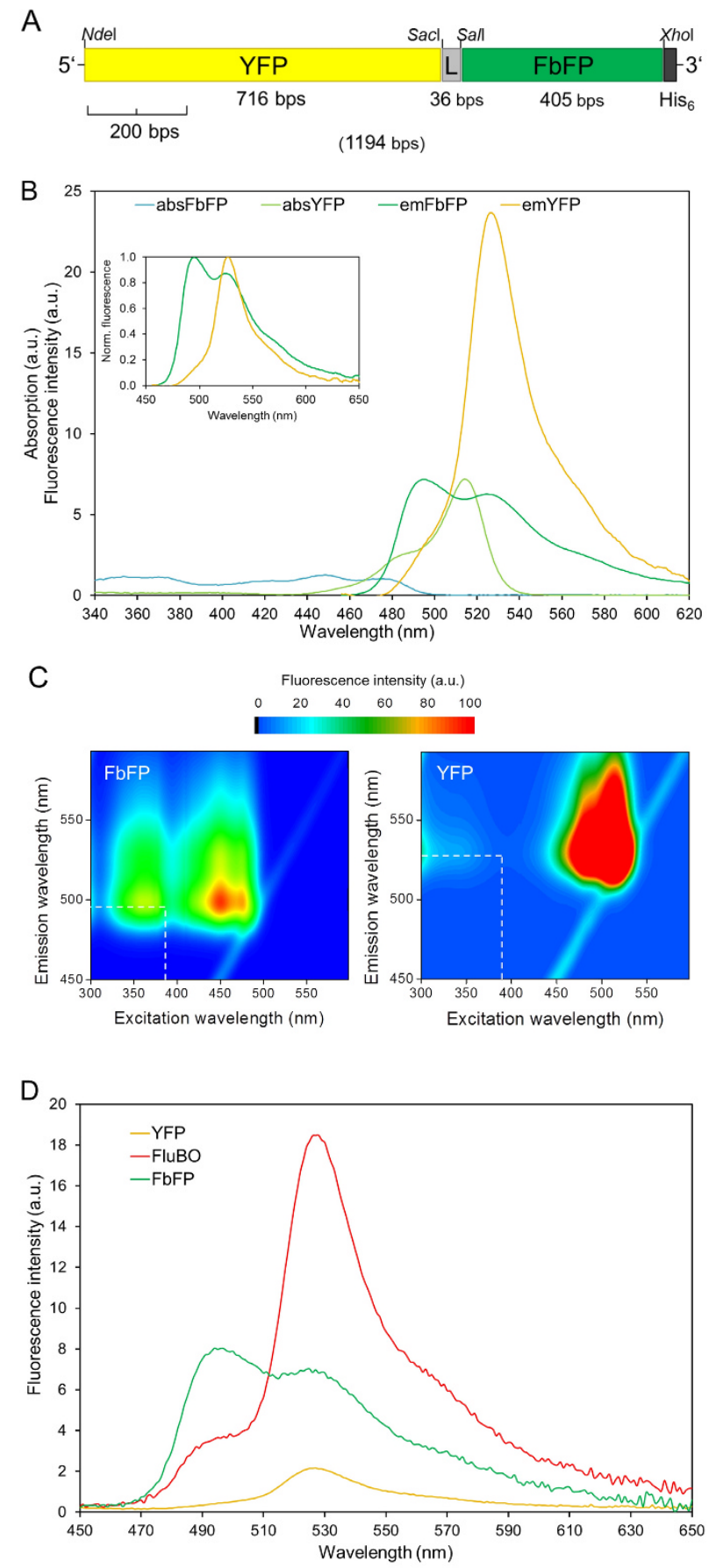

Figure 1 Design and spectral properties of the FRET-based oxygen biosensor FluBO. (A) Genetic construction of the 1194-bp biosensor gene. The YFP encoding DNA fragment ( $5^{\prime}$-end) was fused to the FbFP encoding fragment ( $3^{\prime}$-end) by a 36-bp oligonucleotide which codes for a peptide linker $(\mathrm{L})$ containing a thrombin cleavage site. The restriction endonuclease cleavage sites used for the construction of the biosensor gene are shown. (B) Absorption and fluorescence spectra $\left(\lambda_{\text {exc }}=440 \mathrm{~nm}\right)$ of FbFP and YFP in phosphate buffer $(\mathrm{pH} 8.0,10 \mathrm{mM} \mathrm{NaCl})$. Absorption spectra are given considering the ratio of the absorption maxima of YFP $(514 \mathrm{~nm})$ and FbFP (450 nm) that corresponds to the ratio of the respective extinction coefficients (YFP [35]: 72,000 $\mathrm{cm}^{-1} \mathrm{M}^{-1}$, FbFP: $12,500 \mathrm{~cm}^{-1} \mathrm{M}^{-1}$ ). Accordingly, the fluorescence spectra were adjusted considering the ratio of the respective fluorescence quantum yields (YFP [35]: 0.76, FbFP: 0.39). The inset additionally shows the corresponding normalized FbFP and YFP fluorescence spectra. (C) Two-dimensional wavelength scans of FbFP and YFP. Purified proteins were adjusted to an absorption of $0.1\left(\lambda_{\text {FbFP }}=450 \mathrm{~nm} ; \lambda_{\text {YFP }}=514 \mathrm{~nm}\right)$ and fluorescence emission spectra (450 nm to $595 \mathrm{~nm}$ ) were recorded upon increasing excitation wavelengths from $300 \mathrm{~nm}$ to $600 \mathrm{~nm}$. The optimal excitation wavelength, where FbFP but not YFP showed bright fluorescence, is marked by dashed lines. (D) Emission spectrum of purified FluBO in comparison to the spectra of donor and acceptor fluorescent proteins FbFP and YFP at $\lambda_{\text {exc }}=380 \mathrm{~nm}$; a.u.: arbitrary units. The three proteins were used in equimolar concentrations (approximately $1.5 \mu \mathrm{M}$ ) as judged by the overlap of their absorption spectra in the region > $500 \mathrm{~nm}$ (YFP and FluBO) and 320 to $380 \mathrm{~nm}$ (FbFP and FluBO). 


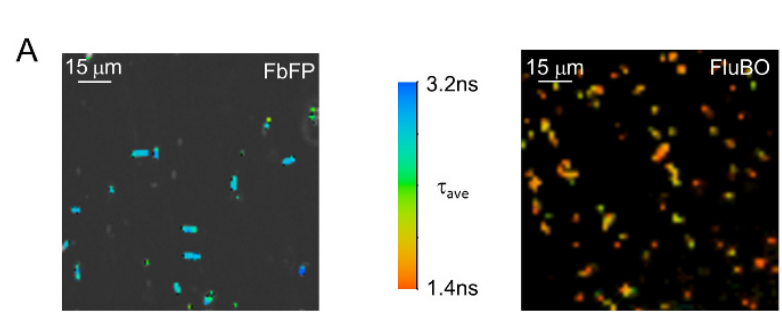

B

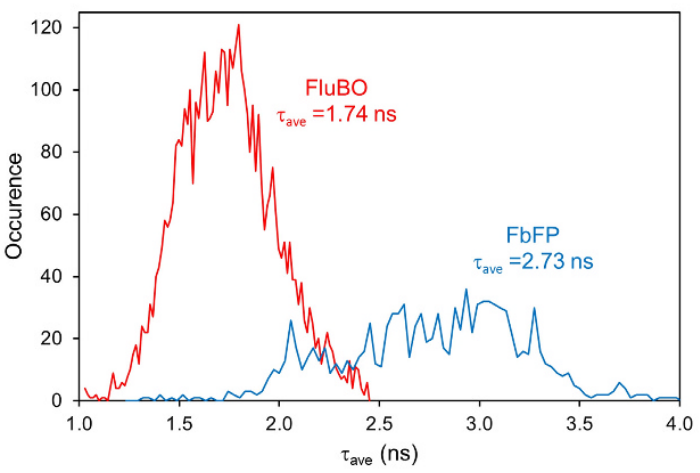

C

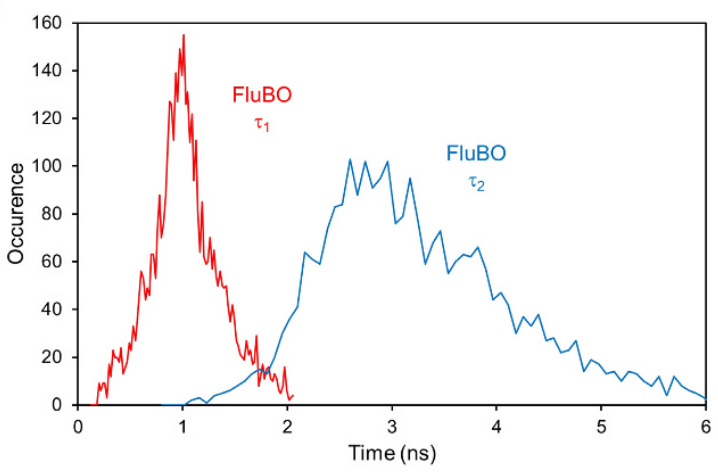

Figure 2 In vivo analysis of FluBO fluorescence lifetime. (A) FLIM pictures of E. coli cells expressing either FbFP or FluBO. Cells were cultivated until they reached the stationary growth phase. Fluorescence was observed at the maximum of FbFP $\left(\lambda_{\text {obs }}<500 \mathrm{~nm} ; \lambda_{\text {exc }}=760 \mathrm{~nm}\right)$. Two-photon excitation of FluBO fluorescence was tested at different wavelengths and was found best at $760 \mathrm{~nm}$, corresponding the doubled wavelength of the normal one-photon excitation optimum (380 nm, see Figure 1D). The figure shows the fluorescence lifetimes ( $\tau_{\text {ave }}$ ) of FbFP (left panel) and FluBO (right panel) in living E. coli cells. (B, C) Analysis of the fluorescence decays of FluBO and FbFP expressed in E. coli under aerobic conditions (data are derived from images shown in Figure 2A). The fluorescence decay of FbFP was satisfactorily analyzed with a monoexponential decay function $\left(F(t)=a \cdot \exp \left(-\tau_{1} / t\right) ; \tau_{\text {ave }}=\tau=2.73 \mathrm{~ns}\right.$ ) while the fluorescence decay of FluBO needed a biexponential decay for correct description $\left(F(t)=a_{1} \cdot \exp \left(-\tau_{1} / t\right)+a_{2} \cdot \exp \left(-\tau_{2} / t\right) ; \tau_{\text {ave }}=\left(a_{1} \cdot \tau_{1}+a_{2} \cdot \tau_{2}\right) /\left(a_{1}+a_{2}\right)=1.74 \mathrm{~ns}\right) ; \tau_{1}=1.03 \mathrm{~ns}, \tau_{2}=2.72 \mathrm{~ns}$. Due to FRET, the average fluorescence lifetime $\tau_{\text {ave }}$ of FbFP in FluBO is reduced by 0.99 ns compared to FbFP alone. Using the average lifetimes of FbFP and FluBO and

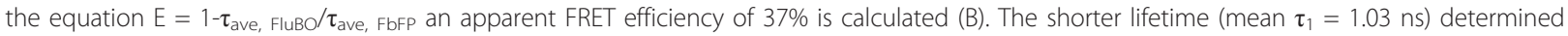
form detailed analysis of the FluBO-FLIM experiments exhibited a major amplitude of $67 \%$. Hence, a FRET efficiency of approximately $62 \%$ within the FluBO proteins that undergo FRET can be deduced from $\tau_{1}\left(E=1-\tau_{1} / \tau_{\text {ave, FbFP }}\right.$. The remaining $33 \%$ of FluBO molecules behave like single isolated FbFP molecules with a long lifetime of 2.72 ns (C).

observed at the FbFP fluorescence maximum. Reduction of in vivo fluorescence lifetimes of plain FbFP (monoexponential analysis, $\tau_{\text {ave }}=2.73 \mathrm{~ns}$ ) in comparison to FbFP fused to the FRET acceptor domain YFP (biexponential analysis, $\tau_{\text {ave }}=1.74 \mathrm{~ns}$ ) (Figure 2A, B) again revealed efficient energy transfer between the donor and the acceptor domain of FluBO with a difference in fluorescence lifetime $(\Delta \tau)$ of $0.99 \mathrm{~ns}$ under aerobic conditions. The apparent FRET efficiency $(E)$ is $37 \%$ using $\tau_{\text {ave }}$
(Figure 2B). A detailed analysis of the FluBO-FLIM experiments in aerated $E$. coli cells revealed that two exponential functions are necessary to describe the observed fluorescence decay behavior satisfactorily (Figure $2 \mathrm{C}$ ). Here, the longer fluorescence lifetime is very similar to that of FbFP alone. The other, shorter lifetime (mean $\tau_{1}=1.03 \mathrm{~ns}$ ) exhibiting the major amplitude (mean relative amplitude of 67\%) represents the FRET process (donor to acceptor energy transfer). Hence, a 
FRET efficiency of approximately $62 \%$ within the FluBO proteins can be deduced from $\tau_{1}$. In comparison, the original FRET donor CFP exhibits a similar fluorescence lifetime with a $\tau_{\text {ave }}$ of 2.23 ns whereas FRET-mediated quenching led to an average fluorescence lifetime of $1.67 \mathrm{~ns}$ within a YFP-CFP fusion $(\Delta \tau=0.56 \mathrm{~ns})$ and an apparent efficiency $E$ of $25.0 \%$ [35].

Afterwards, we studied the oxygen-dependent fluorescence response of FluBO in E. coli cells during batch cultivation (Figure 3A). To this end, the BioLector microbioreactor system (m2p-labs, Aachen, Germany) was used, which provides quantitative online data of the cell density via scattered light intensity (black curve) and dissolved oxygen tension (DOT; blue curve) as described previously $[30,36,37]$. In parallel, intracellular oxygen values of $E$. coli cells expressing FluBO were permanently recorded during cultivation by measuring the donor fluorescence at $492 \mathrm{~nm}$ (FbFP, green) and the acceptor fluorescence at $532 \mathrm{~nm}$ (YFP, yellow) with an excitation wavelength of $380 \mathrm{~nm}$. After approximately three hours of cultivation, FluBO expression was autoinduced as indicated by rapidly increasing fluorescence intensities of both FP domains. After five hours $\left(t_{o}\right)$, high respiratory activity of exponentially growing bacteria caused a drastic limitation of $\mathrm{O}_{2}$ in the growth medium (DOT $\leq 5 \%$ ). During this phase of growth, concomitant intracellular oxygen depletion was reflected by a weak increase of YFP fluorescence due to permanently increasing cell numbers and accumulated biosensor protein at low $\mathrm{O}_{2}$ concentrations. After 13 hours of cell cultivation, the change of carbon source from glycerol to the overflow metabolite acetate, produced in the first phase of the fermentation, led to a reduced oxygen consumption of $E$. coli cells. Thus, the diauxic growth temporarily elevated the intracellular oxygen value (Figure $3 \mathrm{~A}, \mathrm{t}_{1}-\mathrm{t}_{2}$ ) which could be monitored instantly by an increasing YFP fluorescence accompanied by FRET-based decrease of FbFP fluorescence. Consequently, reduced cell respiration led to a transient shift of DOT to a maximal level of $11 \%$. Finally, the DOT level rapidly increased after 16.0 hours when the culture entered the stationary growth phase $\left(t_{3}\right)$. As expected, this final change of the oxygen concentration also caused an immediate increase of YFP fluorescence accompanied by a decrease of FbFP fluorescence.

The YFP-FbFP fusion construct FluBO represents a FRET pair and thus provides a fixed stoichiometry of the two FP domains. Hence, the ratio of observed YFP and FbFP fluorescence emissions directly reflects the change of cellular oxygen concentration (Figure 3B). Remarkably, extra- and intracellular $\mathrm{O}_{2}$ values are directly correlated and developed cooperatively during cell cultivation. For instance, low DOT which occurred in the late logarithmic growth phase (Figure $3 \mathrm{~A}, \mathrm{~B} ; \mathrm{t}_{2}-\mathrm{t}_{3}$ ) resulted in an almost constant YFP-to-FbFP fluorescence ratio (Figure 3B) until the molecular oxygen concentration increased due to the physiological adaptation of the bacteria during the stationary growth phase. Here, the change of intracellular oxygen availability is directly reflected by an increasing FluBO fluorescence ratio. The ratiometric change of donor and acceptor fluorescence is caused by $\mathrm{O}_{2}$-mediated maturation of the YFP chromophore resulting in dominant YFP fluorescence and a FRET-dependent quenching of FbFP fluorescence (Figure 3A, B). However, bacterial proteins exhibiting an intrinsic fluorescence may affect the biosensor-based oxygen readout. Therefore, an E. coli strain carrying the corresponding empty expression vector was cultivated under the same conditions and the ratio of cellmediated background fluorescence was analyzed at identical wavelengths (Figure 3C). Here, an only marginal change of the emission ratio was detected, which was clearly independent of the DOT level.

\section{In vivo calibration of the fluorescent protein-based oxygen biosensor}

The results presented so far clearly demonstrate that the development of DOT directly correlates with intracellular oxygen levels basically allowing the in vivo calibration of the FRET biosensor within the used E. coli test system. However, the emission ratio of FluBO at a certain time point of cell growth is modulated by the availability of oxygen as well as by the time needed for FluBO synthesis and YFP chromophore formation. Furthermore, oxygen-dependent maturation of the YFP chromophore is an irreversible process. Therefore, repeating changes from sufficient to deficient oxygen concentrations as occurring during batch cultivation of $E$. coli indeed resulted in the inhibition of YFP chromophore maturation and thus led to almost constant but stepwise increased cyan-to-yellow fluorescence ratios under anaerobic conditions (Figure 3B). For that reason, we subsequently considered the change of FluBO fluorescence ratio over time $(\mathrm{d}($ ratio $) / \mathrm{d}(\mathrm{t}))$ as a measure for fluctuating intracellular $\mathrm{O}_{2}$ concentration. To accurately calibrate FluBO in vivo, E. coli cells were batch cultured to the mid-logarithmic growth phase (that is, to oxygen limited conditions) and, subsequently, DOT was temporarily increased twice. As shown in Figure 4A the change of FluBO emission ratio over time directly corresponded to the respective DOT levels. Remarkably, with the onset of repeated $\mathrm{O}_{2}$ deprivation in the medium, lowering of intracellular oxygen level led to a retarded YFP chromophore maturation, which in turn was directly reflected by a decreasing (d (ratio)/d (t)) value of FluBO fluorescence. Hence, this new method also allows the indirect detection of decreasing oxygen levels in living cells. Finally, a linear oxygen calibration curve could be derived from the E. coli in vivo fluorescence data (Figure 4B) covering a DOT level from 0 to $33 \%$ corresponding to a molecular $\mathrm{O}_{2}$ concentration up to $0.08 \mathrm{mmol} / \mathrm{l}$. 

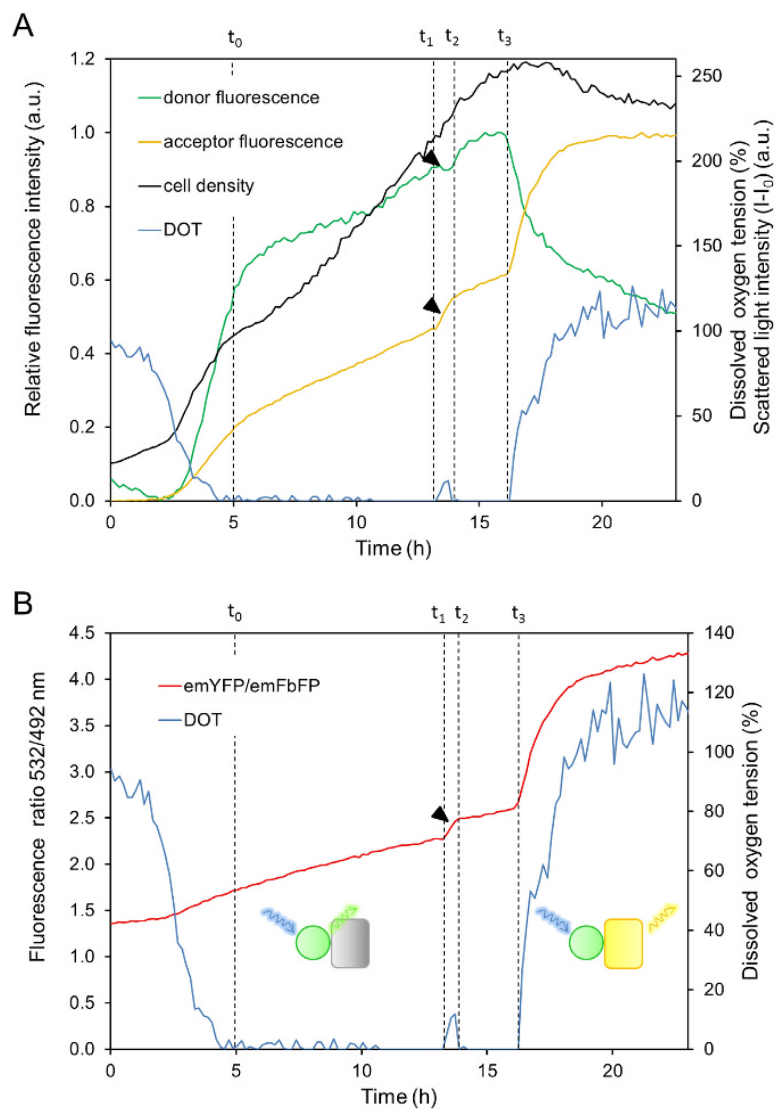

C

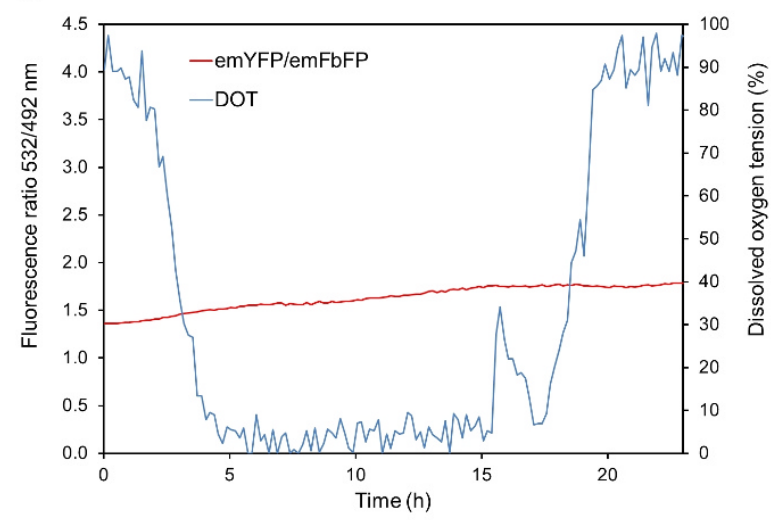

Figure 3 Determination of intracellular oxygen concentration in E. coli. E. coli cells expressing FluBO were batch cultured in Overnight Express ${ }^{\mathrm{TM}}$ Instant TB medium in 48-well flowerplates using the microcultivation system BioLector. The development of biomass (black), dissolved oxygen tension (blue) and FP-mediated fluorescence (FbFP: green, YFP: yellow) was online-monitored in each well of the flowerplate. Cell density was monitored by scattered light (I-lo) at an excitation wavelength of $620 \mathrm{~nm}$ (a.u.: arbitrary units). Time points of changing DOT of the growth medium are labeled ( $t_{0}$ to $t_{3}$ ). Arrowheads indicate changes of donor and acceptor fluorescence which is caused by a temporary shift of DOT during the logarithmic growth phase. (A) Fluorescence emission of donor (FbFP) and acceptor (YFP) was recorded at $492 \mathrm{~nm}$ and $532 \mathrm{~nm}$, respectively, with an excitation wavelength of $380 \mathrm{~nm}$. (B) Development of cyan-to-yellow fluorescent ratio (emYFP/emFbFP) of the FRET-based oxygen biosensor is shown in red. The pictograms symbolize the state of fluorescence activity of the FbFP donor domain (circle) and YFP acceptor domain (rectangle). (C) Background fluorescence of E. coli during cell growth. E. coli cells carrying the empty expression vector were batch cultured in Overnight Express ${ }^{\mathrm{TM}}$ Instant TB medium in 48-well flowerplates using the BioLector. Dissolved oxygen tension is shown in blue. According to emFbFP and emYFP, cell-dependent background fluorescence was online recorded at $492 \mathrm{~nm}$ and $532 \mathrm{~nm}$ that, respectively, at an excitation wavelength of $380 \mathrm{~nm}$ and the fluorescence ratio was calculated (red curve). All parallel fermentation experiments in the microwells were performed in triplicates. These results were in excellent agreement (see Additional File 4). Maximum time shifts of 10 minutes occurred due to unavoidable slight differences of cell density at the beginning of the cultivation. Therefore, a representative set of data from these three parallel independent measurements instead of the corresponding mean values is shown in the figures. 


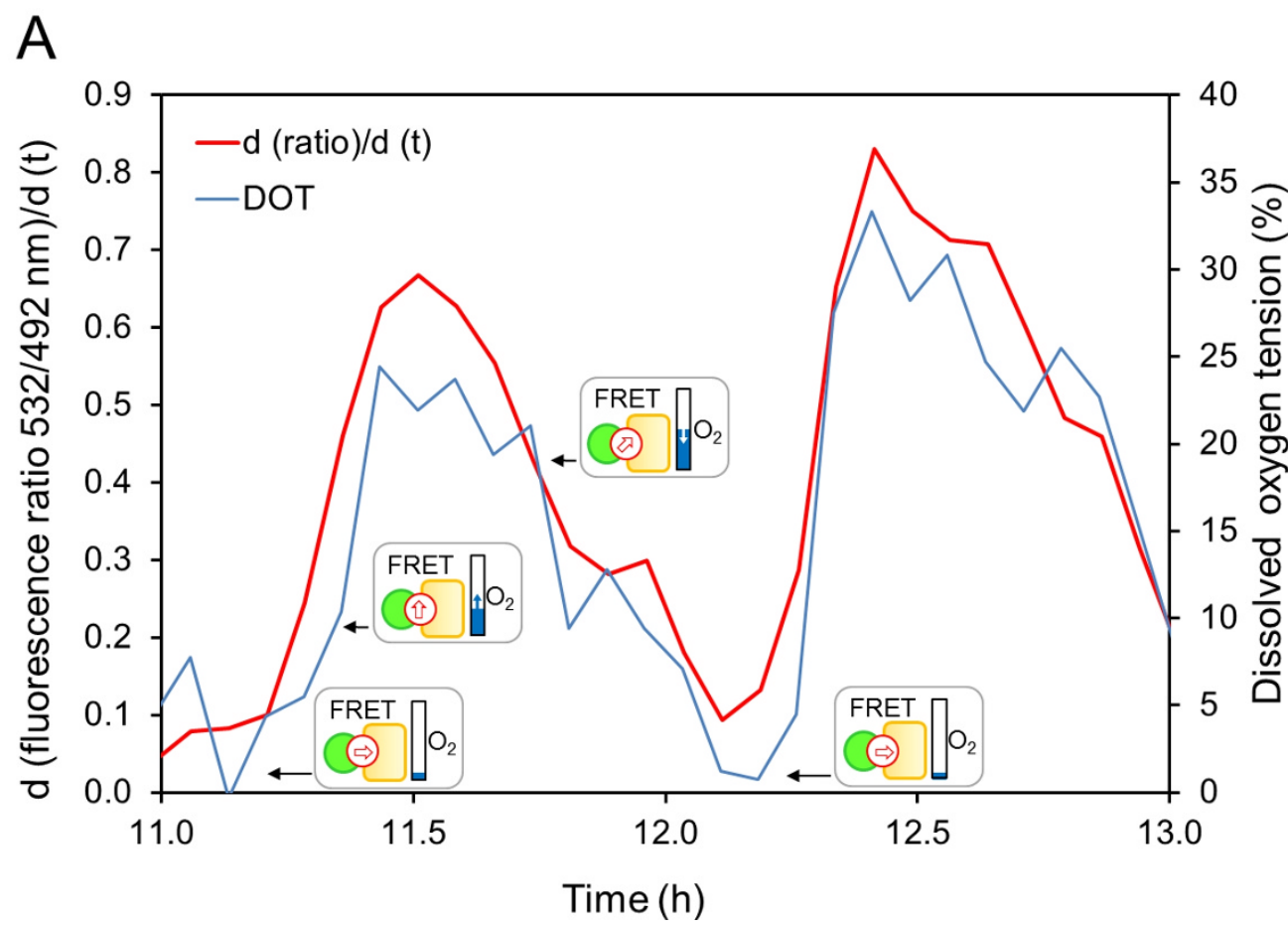

B

Dissolved oxygen tension (\%)

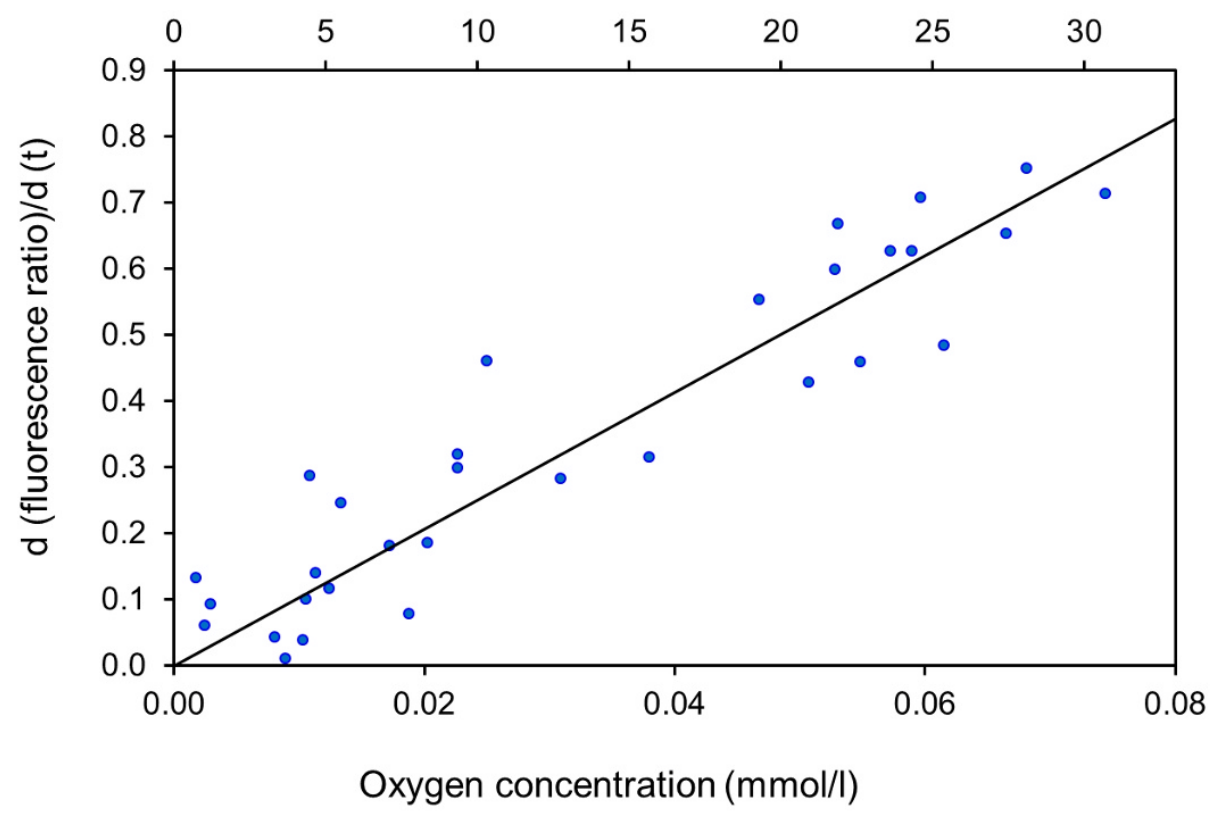

Figure 4 Calibration of FluBO for quantitative determination of intracellular oxygen. (A) During the logarithmic growth of E. coli cells, oxygen limitation was temporarily relieved twice by increasing the shaking frequency. The fitting curve of the time derivation of cyan-to-yellow fluorescence ratio (red) was drawn as described in the Methods. The pictograms symbolize the velocity of change of FRET ratio in dependence on cellular oxygen values: $\Rightarrow$, no change; $\widehat{\imath}$, fast increase; $\pi$, slow increase. (B) The same data have been used to generate the calibration curve. In the used medium the value of $100 \%$ DOT corresponds to an oxygen concentration of $0.24 \mathrm{mmol} / \mathrm{l}$ at $30^{\circ} \mathrm{C}$ and an atmospheric pressure of $100 \mathrm{kPa}$. All parallel fermentation experiments in the microwells were performed in triplicates. The figures show a representative set of data. 


\section{Discussion}

\section{Characteristics of the oxygen biosensor FluBO}

Here, we describe for the first time the development of a genetically encoded FRET-based biosensor, allowing the determination of molecular oxygen inside live cells. The biosensor FluBO consists of the cyan fluorescing flavoprotein FbFP and the yellow fluorescing GFP derivative YFP as respective FRET donor and acceptor domains. Here, the oxygen demand for chromophore formation of YFP, but not of FbFP is the basis for the detection of cellular oxygen changes via the $\mathrm{O}_{2}$-dependent alteration of FRET efficiency. At atmospheric levels of $\mathrm{O}_{2}$, YFP chromophore maturation leads to efficient FRET coupling causing an efficient quenching of FbFP fluorescence and a dominant YFP fluorescence. In contrast, the absence of molecular oxygen prevents YFP chromophore formation and thus immature YFP has no effect on the fluorescence of the FbFP donor domain due to the loss of FRET coupling. To evaluate the in vivo functionality of FluBO, we extensively analyzed the biosensor in $E$. coli cells during batch cultivation. Parallel online detection of extra- and intracellular oxygen levels using an oxygen sensitive optode and the genetically encoded biosensor revealed a clear correlation between $\mathrm{O}_{2}$ levels outside and inside the cells.

\section{Calibration of FluBO}

Development of DOT directly correlates with intracellular $\mathrm{O}_{2}$ levels hence allowing the in vivo calibration of the FRET biosensor within the used $E$. coli test system. The resulting oxygen calibration curve was derived from the FluBO (d (ratio)/d (t)) values that correspond to defined concentrations of extracellular oxygen.

Generally, novel intramolecular FRET-based biosensors are pre-calibrated in vitro and subsequently transferred into the living system of interest [38-41]. However, in contrast to other FP-based biosensors which allow the quantification of a certain metabolite, in vitro calibration of FluBO is not an easy task. Accurate pre-calibration requires biosensor protein, which was expressed and subsequently purified under anaerobic conditions. In addition, in vitro calibration has to be conducted using the gaseous substrate $\mathrm{O}_{2}$ in an otherwise oxygen-free atmosphere. Furthermore, biosensor pre-calibration basically does not take into account that differences in non-specific interactions between a molecule (that is, the respective biosensor) and its immediate surrounding (for example, a buffer in comparison to the interior of a living cell) can greatly influence the equilibrium and rate of the respective reaction $[42,43]$. For that reason, the use of pre-calibrated reference probes does not inevitably lead to accurate in vivo calibration of a novel biosensor. Finally, as discussed below, the level of biosensor expression and, to a minor extent, its stability (or turnover) in the respective host cell rather than the cellular growth rate most probably represents the major bottleneck for functionality of a genetically encoded biosensor. However, these factors significantly depend on the individual properties of a biological system and might even vary within a particular cell depending on growth conditions and/or environmental parameters. Additionally, ratio of extra- and intracellular oxygen concentrations in different organisms might vary in dependence on $\mathrm{O}_{2}$ consumption, which in turn can also be affected by individual parameters, such as variable metabolic activities and cell organization as well as heterogeneity of oxygen diffusion within cellular compartments and tissues. Consequently, the intracellular $\mathrm{O}_{2}$ concentration inside $E$. coli cells is probably much lower than in their surrounding medium (reflected by DOT). Therefore, the applicability of an intracellular biosensor as a quantitative probe needs to be evaluated and calibrated separately for each organism and condition to achieve reliable data.

\section{Environmental sensitivity of FluBO}

Most of the YFP derivatives described in the literature exhibit a distinct $\mathrm{pH}$ sensitivity with $\mathrm{pK}_{\mathrm{a}}$ values ranging from 6.9 (EYFP) to 5.7 (mCitrine) [28]. Using YFP as a FRET receptor domain, therefore, ideally requires a constant fluorescence brightness which is not affected by intracellular changes of $\mathrm{pH}$. To quantitatively interpret oxygen measurements with FluBO, we utilized an EYFP variant distributed by Clontech-Takara (details are described within the Methods section and the corresponding EYFP sequence is shown in Additional file 1). Analysis of $\mathrm{pH}$ sensitivity demonstrated that this YFP derivative exhibits a remarkable $\mathrm{pH}$ resistance $\left(\mathrm{pK}_{\mathrm{a}}=5.2\right.$; Additional file 3 ). Thus, FluBO-based oxygen determination is not affected within the physiological $\mathrm{pH}$ range. In addition, the early YFP variants were also sensitive towards chloride [28]. To further rule out that chloride ions influenced the FluBO oxygen response in vivo, we also determined $\mathrm{Cl}^{-}$ sensitivity of the used EYFP. We could demonstrate that YFP fluorescence intensity remained constant in the presence of chloride concentrations up to $100 \mathrm{mM}$ (data not shown). This result clearly indicates that FluBO is also insensitive to changes of chloride-ion concentrations in the physiological relevant range.

\section{Limitations of FluBO}

Although FluBO is a useful molecular probe, some aspects must be considered to allow online-detection of intracellular oxygen: First, as mentioned above, oxygendependent chromophore formation is an irreversible process. Consequently, this biosensor indeed allows analyzing changes from low to high oxygen concentrations but it cannot directly be used to detect reduction of 
oxygen levels via its change of intramolecular FRET efficiency. Therefore, we analyzed alterations from sufficient to deficient oxygen levels by observing the total changes of dual emission ratio over time. By using this method, recurring oxygen limitation was reflected by a stagnating alteration of YFP-to-FbFP fluorescence ratio (as for example, visible in Figure 3B between t2 and t3) and in turn by decreasing (d (ratio)/d (t)) values (Figure 4A). Furthermore, it should be emphasized that due to the irreversibility of $\mathrm{O}_{2}$-mediated YFP chromophore formation repeated changes or a long-lasting increase of intracellular oxygen level can be determined only under specific conditions. Namely, the degradation of oxidized FluBO and the production rate of non-oxidized FluBO need to form an equilibrium ensuring a continuous and sufficient supply of biosensor molecules which did not 'sense' oxygen before. It, therefore, remains to be experimentally demonstrated how FluBO behaves in cells exhibiting significantly lower metabolic activity than $E$. coli. Nevertheless, we expect the FluBO (d (ratio)/d (t)) value to represent a valid indication of intracellular oxygen levels in a large variety of different (micro)organisms. To this end, we are currently analyzing the applicability of FbFP and the FbFP-based biosensor FluBO in various prokaryotic and eukaryotic including mammalian cell lines.

\section{Alternative in vivo $\mathrm{O}_{2}$ sensors}

Monitoring the heterogeneity and development of intracellular oxygen levels with high spatio-temporal resolution requires a molecular $\mathrm{O}_{2}$ biosensor that exhibits some important properties. For example, the ideal oxygen indicator must be either uniformly distributed or, alternatively, can be specifically targeted within a cell or tissue of interest. For quantitative in vivo $\mathrm{O}_{2}$ determination, the biosensor has to respond rapidly, reliably and reversibly to the full range of physiological oxygen concentrations.

In the last decade, different fluorescent dyes and proteins have been developed, which can be used as sensors for intracellular oxygen measurements.

(1) Various fluorescence and phosphorescence based $\mathrm{O}_{2}$-sensitive probes, including the complex platinum(II)porphyrin dyes are widely used for imaging molecular oxygen in living organisms [17-19]. These probes exhibit a selective and reversible response to $\mathrm{O}_{2}$ within the full physiological oxygen range (0 to $250 \mu \mathrm{M})$ combined with optimal photophysical properties (for example, high fluorescence brightness). Nevertheless, in comparison to genetically encoded sensors intracellular loading of these dyes appears to be relatively inefficient, slow and, furthermore, they cannot be generally targeted to specific cells or intracellular compartments $[19,44,45]$.
Consequently, intracellular visualization of $\mathrm{O}_{2}$ with high spatio-temporal resolution is limited.

(2) Alternatively, GFP can be utilized as a reporter gene, whose expression is under control of an oxygen sensitive regulator within a certain organism. For example, in E. coli promoters $\mathrm{P}_{\text {nar }}$ (controlling nitrate reductase expression) and $\mathrm{P}_{\text {rpos }}$ (controlling the expression of the stress response sigma factor $\sigma^{\mathrm{S}}$ ), which are activated under microaerobic and anaerobic conditions, were applied to monitor oxygen limitations [20,21]. Although this technique is potentially capable of analyzing limited oxygen supply and differences in population heterogeneities, this biosensor architecture can only be applied for specific host organisms. Furthermore, regulators are often part of a higher-order regulatory network and thus might not solely respond to oxygen deprivation. Beside oxygen sensitive GFP reporter gene constructs, Takahashi and coworkers described an exceptional technique that also allows GFP-dependent in vivo oxygen determination [22-24]. This approach makes use of the oxygen sensitive red-shifted fluorescence of GFP that occurs after photoactivation with blue light [46]. However, since the magnitude of the observed red shift is abruptly increased with oxygen concentrations $<1 \%$, this technique can only be applied for measuring low intracellular oxygen levels. Furthermore, the basic mechanism of GFP photoactivation of red fluorescence as well as the oxygen-dependent recovery to green fluorescence is not fully understood yet. Finally, it is worth mentioning that both of the described strategies, using GFP either as 'passive' or 'active' oxygen biosensor, inherently suffer from impaired chromophore formation under oxygen limited conditions. This fact makes FluBO the first fluorescent protein-based biosensor enabling exact and reliably determination of molecular oxygen.

\section{Conclusions}

Accurate determination of intracellular oxygen and analysis of changing $\mathrm{O}_{2}$ levels inside living cells and tissues is of the utmost importance in gaining new insights into complex (patho)physiological processes associated with oxygen deprivation, including invasion and persistence of pathogens or tumor development. Our results show that FluBO can be used as a ratiometric biosensor for molecular oxygen that changes the emission maxima of the donor and acceptor domain as a function of $\mathrm{O}_{2}$ availability and its sensitivity is within the physiological range relevant for bacteria as well as for eukaryotes, including mammalian and human cells [2]. The oxygen modulated FRET-coupling allows the non-invasive readout of cellular $\mathrm{O}_{2}$ levels as well as the online analysis of its changes with high spatial and temporal resolution by fluorescence spectrometry and FLIM. Thus, the 
observation of cellular oxygen levels in any cellular compartment using genetically encoded FRET biosensors will offer a novel rational approach to understand hypoxia-dependent processes in biological, biomedical and biotechnological processes.

\section{Methods}

\section{Bacteria and growth conditions}

E. coli strain DH5 $\alpha$ [47] was used for DNA cloning of the expression vectors encoding the fluorescent reporter proteins FbFP and YFP, as well as the biosensor FluBO. E. coli strain BL21(DE3) (Novagen, distributed by Merck KGaA, Darmstadt, Germany) was used for expression of the fluorescent proteins and FRET-based biosensor FluBO. For protein expression and purification, bacterial cells were grown either in autoinduction TB medium consisting of $5 \mathrm{~g} / \mathrm{l}$ glycerol, $12 \mathrm{~g} / \mathrm{l}$ tryptone, $24 \mathrm{~g} / \mathrm{l}$ yeast extract, $2.32 \mathrm{~g} / \mathrm{l} \mathrm{KH}_{2} \mathrm{PO}_{4}, 12.5 \mathrm{~g} / \mathrm{l} \mathrm{K}_{2} \mathrm{HPO}_{4}(\mathrm{pH}$ 7.2), lactose $2 \mathrm{~g} / \mathrm{l}$, glucose $0.5 \mathrm{~g} / \mathrm{l}$ (for FbFP expression and purification) or in LB medium consisting of $10 \mathrm{~g} / \mathrm{l}$ tryptone, $5 \mathrm{~g} / \mathrm{l}$ yeast extract, $10 \mathrm{~g} / \mathrm{l} \mathrm{NaCl}$ (for YFP expression and purification). BioLector cultivation experiments were conducted in Overnight Express ${ }^{\mathrm{TM}}$ Instant $\mathrm{TB}$ medium (Novagen) as described below. All media were supplemented with $50 \mu \mathrm{g} / \mathrm{l}$ kanamycin to maintain expression vector pRhotHi-2.

\section{Construction of FP expression vectors}

The construction of expression vector pRhotHi-2-YFP encoding the enhanced yellow fluorescent protein YFP (available from Clontech-Takara Bio Europe, Saint-en-laye, France) and pRhotHi-2-FbFP encoding EcFbFP (GenBank number: ABN71355; commercially available as evoglowBs2, evocatal GmbH, Düsseldorf, Germany) was described previously [31]. The YFP variant used in this study contains four amino acid substitutions previously published as GFP$10 \mathrm{C}$ [48], as well as the substitution H231L and the insertion of valine behind the first amino acid. The expression vector pRhotHi-2-FluBO harboring the FRET-based oxygen biosensor encoding gene was constructed using a synthetic FluBO gene (Eurofins MWG Operon, Ebersberg, Germany) whose DNA sequence is shown in Additional file 1 . The biosensor gene was cloned into the NdeI and XhoI restriction sites of pRhotHi-2 [49] and recombinant FP genes were confirmed by DNA sequencing. The pRhotHi-2-FluBO vector will soon be commercially available (evocatal GmbH, Düsseldorf, Germany).

\section{Protein expression and purification}

E. coli BL21(DE3) cells carrying the expression plasmids pRhotHi-2-FbFP, pRhotHi-2-YFP and pRhotHi-2-FluBO, respectively, were grown until the cultures reached an optical density of approximately 0.5 at $580 \mathrm{~nm}$. Protein expression was then induced by adding $1 \mathrm{mM}$ isopropyl- $\beta$-D-thiogalactopyranoside (IPTG) to the culture medium. Recombinant oxygen biosensor FluBO, YFP and FbFP were purified as $\mathrm{His}_{6}$-tagged proteins from E. coli BL21(DE3) after $16 \mathrm{~h}$ of expression at $37^{\circ} \mathrm{C}$. Purification was carried out by using HisTag Superflow Hightrap gravityFlow cartridges (Qiagen, Hilden, Germany) and peristaltic pump P-1 (GE Healthcare Europe $\mathrm{GmbH}$, Munich, Germany) under standard operation conditions as described by the manufacturers. The purified proteins were stored at $4^{\circ} \mathrm{C}$ in protein storage solution containing $10 \mathrm{mM} \mathrm{NaCl}, 10 \mathrm{mM} \mathrm{NaH}_{2} \mathrm{PO}_{4}, \mathrm{pH}$ 8.0.

\section{Spectral analysis and fluorometry}

The absorption and fluorescence properties of FbFP, YFP and FluBO were determined in $10 \mathrm{mM} \mathrm{NaCl}$ and $10 \mathrm{mM}$ sodium phosphate buffer $\mathrm{pH} 8.0$ on a UV-2450 absorption spectrophotometer (Shimadzu Europa $\mathrm{GmbH}$, Duisburg, Germany) and a QM-4 fluorescence spectrophotometer (PTI, Ford, West Sussex, UK), the latter with spectral correction for detector and emission monochromator transmission. The absorption spectra were recorded by scanning from $330 \mathrm{~nm}$ to $620 \mathrm{~nm}$; the emission spectra were recorded at excitation wavelengths of $380 \mathrm{~nm}$ or $440 \mathrm{~nm}$. When emission spectra were measured the maximum absorption of the samples did not exceed 0.15. In the case of excitation/emission (2D) scans, fluorescence excitation was determined from $300 \mathrm{~nm}$ to $600 \mathrm{~nm}$ in $5 \mathrm{~nm}$ steps by using the PerkinElmer LS50B Luminescence Spectrometer (Wellesley, MA, USA) at $22^{\circ} \mathrm{C}$. Here, the fluorescence emission spectra were detected from $450 \mathrm{~nm}$ to $595 \mathrm{~nm}$ wavelength.

\section{Thrombin protease cleavage}

For the thrombin protease mediated cleavage of the FluBO fusion protein, $10 \mu \mathrm{g}$ of purified protein and one unit of thrombin protease (Novagen, distributed by Merck KGaA, Darmstadt, Germany) were diluted in 50 $\mu \mathrm{l}$ thrombin cleavage buffer. After incubation at room temperature for 16 hours an aliquot of $10 \mu \mathrm{l}$ was used for SDS-PAGE analysis to corroborate the cleavage of the YFP-FbFP fusion by thrombin protease. SDS-PAGE gels were prepared using standard methods (NuPAGE, 4 to $12 \%$ Bis-Tris gel $1 \mathrm{~mm}$, Invitrogen, Paisley, UK). After SDS-PAGE separation, proteins were transferred to a PVDF membrane (Bio-Rad Laboratories GmbH, Munich, Germany) by Western blotting (NuPAGE, Invitrogen, Paisley, UK). Subsequently, YFP and FbFP proteins were detected using the respective antibodies (rabbit anti-GFP, BD Biosciences Erembodegem, Belgium; YtvA-specific antiserum). To detect the YFP-FbFP fusion protein FluBO, a His-tag antibody (Anti-His-HRP antibody, Invitrogen, Paisley, UK) was used. Immunodetected proteins were visualized using a CCD camerabased chemiluminescence detection system (Stella 
imaging system with AIDA advanced image data analyzer, Raytest, Straubenhardt, Germany). Fluorescence emission of FPs was determined by using the PerkinElmer LS50B Luminescence Spectrometer at $22^{\circ} \mathrm{C}$ as described above.

\section{Fluorescence lifetime imaging and analysis}

The E. coli cells expressing FbFP, YFP or FluBO were placed in suspension between two glass slides on the stage of an upright fluorescence microscope (BX50WI; Olympus Optical, Tokyo, Japan) and observed through a $60 \times$ water-immersion objective $(\mathrm{NA}=0.9$; Olympus Optical). Fluorescence was excited with ca. 100 fs light pulses $\left(\lambda_{\text {exc }}=760 \mathrm{~nm}\right)$ applied at sufficient intensity to generate two-photon excitation. Light pulses were generated at a frequency of $80 \mathrm{MHz}$ by a mode-locked Titan-Sapphire laser (MaiTai DeepSee; output power > 2W; Newport, Spectra Physics Irvine, CA, USA). The laser light was directed through the lens onto the $E$. coli at reduced power (ca. $5 \mathrm{~mW}$ ) and scanned over the sample using a beam scanner (TILL Photonics, Munich, Germany). Fluorescence was recorded by a photomultiplier (PMT-100, Becker \& Hickl, Berlin, Germany) using appropriate filters for detecting the FbFP fluorescence of FbFP and FluBO (short pass filter $500 \mathrm{~nm} ; \lambda_{\text {obs }}<500$ nm; Omega Optical, Brattleboro, VT, USA) and the YFP fluorescence of YFP and FluBO (band pass filter $535+/$ $13 \mathrm{~nm}$; Omega Optical). Fluorescence lifetime imaging was performed using electronics for time-correlated single photon counting (Simple-Tau 152; Becker \& Hickl) and acquisition software (SPCM8.95; Becker \& Hickl) for time-correlated single-photon counting as described before [50,51]. Lifetime images were analyzed using SPCImage 2.97 (Becker \& Hickl) by fitting mono- or biexponential model functions to the fluorescence decays in every pixel of the image. The program uses an iterative reconvolution of the exponential function with an instrument response function and a least square algorithm for finding parameters of the exponential function for a satisfactory fit.

\section{Characterization of the oxygen biosensor using the BioLector microbioreactor system}

All cultivations were carried out in sterile black 48-well microtiter plates (Flowerplate, m2p-labs, Aachen, Germany) in the microcultivation and on-line monitoring system BioLector (m2p-labs, Aachen, Germany) [36,37]. These microtiter plates allow higher oxygen transfer rates as compared to conventional microtiter plates [52]. Furthermore, each well is equipped with an optode for the determination of DOT (\% air saturation) via fluorescence measurement. The microtiter plates were sealed with sterile gas-permeable adhesive seals (Thermo Scientific, Waltham, MA, USA), ensuring sterile conditions in the wells and allowing good ventilation. The following conditions were applied for all cultivations in the BioLector: temperature $30^{\circ} \mathrm{C}$, total filling volume per well: 600 $\mu \mathrm{l}$, shaking diameter: $3 \mathrm{~mm}$, relative humidity in the incubation chamber: $80 \%$. For all cultivations Overnight Express $^{\mathrm{TM}}$ Instant TB medium (Novagen, distributed by Merck KGaA, Darmstadt, Germany) was used. Cultures were inoculated from pre-cultures to an initial $\mathrm{OD}_{600}$ of 0.1 . Pre-cultures were made in $250 \mathrm{ml}$ flasks under the following conditions: inoculation from a cryoculture to yield an initial $\mathrm{OD}_{600}$ of 0.1 , temperature $37^{\circ} \mathrm{C}$, total filling volume $10 \mathrm{ml}$ of TB medium, shaking diameter 50 $\mathrm{mm}$, shaking frequency $350 \mathrm{rpm}$, growth overnight to stationary phase. For the batch cultivation in the BioLector the shaking frequency was $800 \mathrm{rpm}$. For DOT shift experiments the initial shaking frequency was $600 \mathrm{rpm}$, which was increased to $700 \mathrm{rpm}$ after $11.2 \mathrm{~h}$ and 800 $\mathrm{rpm}$ after $12.2 \mathrm{~h}$. The biomass (I-Io) was measured via scattered light intensity at an excitation wavelength of $620 \mathrm{~nm}$. Donor (FbFP) and acceptor (YFP) fluorescence were excited at $380 \mathrm{~nm}$ and emission was detected at 492 $\mathrm{nm}$ and $532 \mathrm{~nm}$, respectively. DOT was measured at an excitation wavelength of $505 \mathrm{~nm}$ and an emission wavelength of $590 \mathrm{~nm}$. The measuring cycle was 10 minutes for the batch cultivation and 4.5 minutes for the DOT shift experiment. Parallel fermentation experiments in the microwells were performed in triplicates. These results were in excellent agreement. Maximum time shifts of 10 minutes occurred due to unavoidable slight differences of cell density at the beginning of the cultivation. Therefore, a representative set of data from these three parallel independent measurements is shown instead of the corresponding mean values.

\section{Data handling}

For the calibration of the optode-derived DOT signal and the FRET signal we considered the derivation of the YFP-FbFP ratio over the time $(\mathrm{d}($ ratio)/d $(\mathrm{t}))$. In this way, it is possible to also follow decreasing DOT values. To reduce the influence of measuring errors for the calculation of $d($ ratio)/d ( $t$ ) the curves for the FluBO fluorescence ratio were fitted to the raw data. This was done in MATLAB 7.11 (The MathWorks, Inc., Natick, MA, USA) applying a smoothing spline with a smoothing parameter of 0.999. In the used medium the value of $100 \%$ DOT corresponds to an oxygen concentration of $0.24 \mathrm{mmol} / \mathrm{l}$ at $30^{\circ} \mathrm{C}$ and an atmospheric pressure of $100 \mathrm{kPa}$.

\section{Additional material}

Additional file 1: FluBO nucleotide and amino acid sequence. Yellow, YFP sequence; black, linker sequence; green, FbFP sequence; grey, His tag sequence. 
Additional file 2: Fluorescence spectra of FluBO before and after proteolysis with thrombin. Fluorescence spectra of FluBO before and after proteolysis with thrombin. The fluorescence emission spectra of purified FPs $(5 \mu \mathrm{M})$ were recorded at an excitation wavelength of 380 $\mathrm{nm}$. Complete thrombin-catalyzed proteolysis of FluBO was demonstrated by SDS-PAGE (inset), purified FPs were used as standards.

Additional file 3: Sensitivity of used YFP towards $\mathrm{pH}$. The figure shows the normalized YFP fluorescence intensity at increasing $\mathrm{pH}$ values. Purified YFP was adjusted to an absorption of 0.05 . The effect of the $\mathrm{pH}$ value on YFP fluorescence was measured in citric acid/disodium phosphate buffer with $\mathrm{pH}$ values ranging form 4.0 to 7.0. The $\mathrm{pK}_{\mathrm{a}}$ value is marked by doted lines.

Additional file 4: Data from parallel fermentation experiments. The figure shows the individual results from three independent batch cultivation experiments using the BioLector. The development of biomass (black), dissolved oxygen tension (blue) and cyan-to-yellow fluorescent ratio (emYFP/emFbFP) of the FRET-based oxygen biosensor (red) was online-monitored in each well of the flowerplate.

\section{Abbreviations}

ATP: adenosine triphosphate; a.u.: arbitrary units; DOT: dissolved oxygen tension; FbFP: flavin-binding fluorescent protein; FLIM: fluorescence lifetime imaging; FluBO: fluorescent protein-based biosensor for oxygen; FMN: flavin mononucleotide; FP: fluorescent protein; FRET: Förster resonance energy transfer; GFP: green fluorescent protein; IPTG: isopropyl- $\beta-D-$ thiogalactopyranoside; PVDF: polyvinylidene fluoride; QY: quantum yield; SDS-PAGE: sodium dodecyl sulfate polyacrylamide gel electrophoresis; UV ultraviolet; YFP: yellow fluorescent protein.

\section{Acknowledgements}

The authors would like to thank Prof. Dr. Martina Pohl and Dr. Achim Heck for helpful discussion.

\section{Author details}

'Institute of Molecular Enzyme Technology, Heinrich-Heine-University Duesseldorf, Juelich Research Center, Wilhelm-Johnen-Straße, D-52425 Juelich, Germany. ${ }^{2}$ AVT Biochemical Engineering, RWTH Aachen University, Worringerweg 1, D-52074 Aachen, Germany. Institute of Complex SystemsCellular Biophysics (ICS-4), Juelich Research Center, Wilhelm-Johnen-Straße, D-52425 Juelich, Germany.

\section{Authors' contributions}

TD developed the idea for the project and designed the experiments concerning the construction and characterization of the biosensor. JP designed and performed the experiments concerning the construction and characterization of the biosensor. MK and JB designed and performed the BioLector experiments. TG designed and performed the FLIM experiments. $J P, M K, T G, T D$ and JB developed the method for data processing and analysis. TD and K-EJ wrote the paper. All authors read and approve the final manuscript.

\section{Competing interests}

The authors declare competing financial interests. Parts of the results described in this publication have been included into a patent application (Circolone F., Drepper T., Endres S. Heck A., Jaeger K.-E., Potzkei J. (2010); Patent number DE 102010037 001). The oxygen biosensor FluBO will be commercialized by evocatal GmbH, Germany.

Received: 16 December 2011 Accepted: 22 March 2012 Published: 22 March 2012

\section{References}

1. Aragonés J, Fraisl P, Baes M, Carmeliet P: Oxygen sensors at the crossroad of metabolism. Cell Metab 2009, 9:11-22.

2. Ward JPT: Oxygen sensors in context. Biochim Biophys Acta 2008, 1777:1-14
3. Ernst JF, Tielker D: Responses to hypoxia in fungal pathogens. Cell Microbiol 2009, 11:183-190.

4. Hassett DJ, Sutton MD, Schurr MJ, Herr AB, Caldwell CC, Matu JO: Pseudomonas aeruginosa hypoxic or anaerobic biofilm infections within cystic fibrosis airways. Trends Microbiol 2009, 17:130-138.

5. Rustad TR, Sherrid AM, Minch KJ, Sherman DR: Hypoxia: a window into Mycobacterium tuberculosis latency. Cell Microbiol 2009, 11:1151-1159.

6. Schobert M, Jahn D: Anaerobic physiology of Pseudomonas aeruginosa in the cystic fibrosis lung. Int J Med Microbiol 2011, 300:549-556.

7. Schobert M, Tielen P: Contribution of oxygen-limiting conditions to persistent infection of Pseudomonas aeruginosa. Future Microbiol 2010, 5:603-621

8. Eltzschig HK, Carmeliet P: Hypoxia and inflammation. N Engl J Med 2011, 364:656-665

9. Brown JM: Exploiting the hypoxic cancer cell: mechanisms and therapeutic strategies. Mol Med Today 2000, 6:157-162

10. Brown JM: Tumor hypoxia in cancer therapy. Methods Enzymol 2007, 435:297-321.

11. Gordan JD, Simon MC: Hypoxia-inducible factors: central regulators of the tumor phenotype. Curr Opin Genet Dev 2007, 17:71-77.

12. Lu X, Kang Y: Hypoxia and hypoxia-inducible factors: master regulators of metastasis. Clin Cancer Res 2010, 16:5928-5935.

13. Coates JD, Anderson RT: Emerging techniques for anaerobic bioremediation of contaminated environments. Trends Biotechnol 2000, 18:408-412.

14. Karakashev D, Thomsen AB, Angelidaki I: Anaerobic biotechnological approaches for production of liquid energy carriers from biomass. Biotechnol Lett 2007, 29:1005-1012.

15. Löffler FE, Edwards EA: Harnessing microbial activities for environmental cleanup. Curr Opin Biotechnol 2006, 17:274-284.

16. McKinlay JB, Harwood CS: Photobiological production of hydrogen gas as a biofuel. Curr Opin Biotechnol 2010, 21:244-251.

17. Fercher A, O'Riordan TC, Zhdanov AV, Dmitriev Rl, Papkovsky DB: Imaging of cellular oxygen and analysis of metabolic responses of mammalian cells. Methods Mol Biol 2010, 591:257-273.

18. Lee YE, Smith R, Kopelman R: Nanoparticle PEBBLE sensors in live cells and in vivo. Annu Rev Anal Chem (Palo Alto Calif) 2009, 2:57-76.

19. O'Riordan TC, Zhdanov AV, Ponomarev GV, Papkovsky DB: Analysis of intracellular oxygen and metabolic responses of mammalian cells by time-resolved fluorometry. Anal Chem 2007, 79:9414-9419.

20. Delvigne F, Boxus $M$, Ingels $S$, Thonart P: Bioreactor mixing efficiency modulates the activity of a prpoS::GFP reporter gene in E. coli. Microb Cell Fact 2009, 8:15.

21. Garcia JR, Cha HJ, Rao G, Marten MR, Bentley WE: Microbial nar-GFP cell sensors reveal oxygen limitations in highly agitated and aerated laboratory-scale fermentors. Microb Cell Fact 2009, 8:6.

22. Takahashi E, Sato M: Impact of intracellular diffusion of oxygen in hypoxic sensing. Adv Exp Med Biol 2011, 701:301-306.

23. Takahashi E, Takano T, Nomura Y, Okano S, Nakajima O, Sato M: In vivo oxygen imaging using green fluorescent protein. Am J Physiol Cell Physiol 2006, 291:C781-787.

24. Takahashi E, Takano T, Numata A, Hayashi N, Okano S, Nakajima O, Nomura Y, Sato M: Genetic oxygen sensor: GFP as an indicator of intracellular oxygenation. Adv Exp Med Biol 2005, 566:39-44.

25. Li IT, Pham E, Truong K: Protein biosensors based on the principle of fluorescence resonance energy transfer for monitoring cellular dynamics. Biotechnol Lett 2006, 28:1971-1982.

26. Newman RH, Fosbrink MD, Zhang J: Genetically encodable fluorescent biosensors for tracking signaling dynamics in living cells. Chem Rev 2011, 111:3614-3666.

27. Wang H, Nakata E, Hamachi I: Recent progress in strategies for the creation of protein-based fluorescent biosensors. Chembiochem 2009, 10:2560-2577.

28. Shaner NC, Steinbach PA, Tsien RY: A guide to choosing fluorescent proteins. Nat Methods 2005, 2:905-909.

29. Tsien RY: The green fluorescent protein. Annu Rev Biochem 1998 , 67:509-544.

30. Drepper T, Huber R, Heck A, Circolone F, Hillmer AK, Buchs J, Jaeger KE: Flavin mononucleotide-based fluorescent reporter proteins outperform green fluorescent protein-like proteins as quantitative in vivo real-time reporters. Appl Environ Microbiol 2010, 76:5990-5994. 
31. Drepper T, Eggert T, Circolone F, Heck A, Krauss U, Guterl JK, Wendorff M, Losi A, Gärtner W, Jaeger KE: Reporter proteins for in vivo fluorescence without oxygen. Nat Biotechnol 2007, 25:443-445.

32. Frommer WB, Davidson MW, Campbell RE: Genetically encoded biosensors based on engineered fluorescent proteins. Chem Soc Rev 2009, 38:2833-2841

33. Ibraheem A, Campbell RE: Designs and applications of fluorescent protein-based biosensors. Curr Opin Chem Biol 2010, 14:30-36.

34. Palmer AE, Qin Y, Park JG, McCombs JE: Design and application of genetically encoded biosensors. Trends Biotechnol 2011, 29:144-152.

35. Kremers G-J, Goedhart J, van Munster EB, Gadella TWJ Jr: Cyan and yellow super fluorescent proteins with improved brightness, protein folding, and FRET Förster radius. Biochemistry 2006, 45:6570-6580.

36. Kensy F, Zang E, Faulhammer C, Tan RK, Büchs J: Validation of a highthroughput fermentation system based on online monitoring of biomass and fluorescence in continuously shaken microtiter plates. Microb Cell Fact 2009, 8:31

37. Samorski M, Müller-Newen G, Büchs J: Quasi-continuous combined scattered light and fluorescence measurements: a novel measurement technique for shaken microtiter plates. Biotechnol Bioeng 2005, 92:61-68.

38. Arosio D, Ricci F, Marchetti L, Gualdani R, Albertazzi L, Beltram F: Simultaneous intracellular chloride and $\mathrm{pH}$ measurements using a GFPbased sensor. Nat Methods 2010, 7:516-518.

39. Esposito A, Gralle M, Dani MA, Lange D, Wouters FS: pHlameleons: a family of FRET-based protein sensors for quantitative $\mathrm{pH}$ imaging. Biochemistry 2008, 47:13115-13126.

40. Tantama $M$, Hung YP, Yellen G: Imaging intracellular $\mathrm{pH}$ in live cells with a genetically encoded red fluorescent protein sensor. J Am Chem Soc 2011, 133:10034-10037.

41. Yano T, Oku M, Akeyama N, Itoyama A, Yurimoto H, Kuge S, Fujiki Y Sakai Y: A novel fluorescent sensor protein for visualization of redox states in the cytoplasm and in peroxisomes. Mol Cell Biol 2010, 30:3758-3766.

42. Minton AP: How can biochemical reactions within cells differ from those in test tubes? J Cell Sci 2006, 119:2863-2869.

43. van Eunen K, Bouwman J, Daran-Lapujade P, Postmus J, Canelas AB Mensonides FI, Orij R, Tuzun I, van den Brink J, Smits GJ, van Gulik WM, Brul S, Heijnen JJ, de Winde JH, de Mattos MJ, Kettner C, Nielsen J, Westerhoff HV, Bakker BM: Measuring enzyme activities under standardized in vivo-like conditions for systems biology. Febs J 2010, 277:749-760.

44. Dmitriev RI, Ropiak HM, Yashunsky DV, Ponomarev GV, Zhdanov AV Papkovsky DB: Bactenecin 7 peptide fragment as a tool for intracellular delivery of a phosphorescent oxygen sensor. FEBS J 2010, 277:4651-4661

45. Fercher A, Ponomarev GV, Yashunski D, Papkovsky D: Evaluation of the derivates of phosphorescent Pt-coproporphyrin as intracellular oxygensensitive probes. Anal Bioanal Chem 2010, 396:1793-1803.

46. Elowitz MB, Surette MG, Wolf PE, Stock J, Leibler S: Photoactivation turns green fluorescent protein red. Curr Biol 1997, 7:809-812.

47. Hanahan D: Studies on transformation of Escherichia coli with plasmids. $J$ Mol Biol 1983, 166:557-580

48. Ormö M, Cubitt AB, Kallio K, Gross LA, Tsien RY, Remington SJ: Crystal structure of the Aequorea victoria green fluorescent protein. Science 1996 273:1392-1395

49. Katzke N, Arvani S, Bergmann R, Circolone F, Markert A, Svensson V, Jaeger K-E, Heck A, Drepper T: A novel T7 RNA polymerase dependent expression system for high-level protein production in the phototrophic bacterium Rhodobacter capsulatus. Protein Exp Purif 2010, 69:137-146.

50. Gilbert D, Franjic-Würtz C, Funk K, Gensch T, Frings S, Möhrlen F: Differential maturation of chloride homeostasis in primary afferent neurons of the somatosensory system. Int J Dev Neurosci 2007, 25:479-489.

51. Kaneko H, Putzier I, Frings S, Kaupp UB, Gensch T: Chloride accumulation in mammalian olfactory sensory neurons. J Neurosci 2004, 24:7931-7938.

52. Funke M, Diederichs S, Kensy F, Müller C, Büchs J: The baffled microtiter plate: increased oxygen transfer and improved online monitoring in small scale fermentations. Biotechnol Bioeng 2009, 103:1118-1128.

doi:10.1186/1741-7007-10-28

Cite this article as: Potzkei et al:: Real-time determination of intracellular oxygen in bacteria using a genetically encoded FRET-based biosensor. BMC Biology 2012 10:28.

\section{Submit your next manuscript to BioMed Central and take full advantage of:}

- Convenient online submission

- Thorough peer review

- No space constraints or color figure charges

- Immediate publication on acceptance

- Inclusion in PubMed, CAS, Scopus and Google Scholar

- Research which is freely available for redistribution

Submit your manuscript at www.biomedcentral.com/submit 